\title{
Article
}

\section{The impact of cognitive load on processing efficiency and performance effectiveness in anxiety: evidence from event-related potentials and pupillary responses}

Hepsomali, Piril, Hadwin, Julie A, Liversedge, Simon Paul, Degno, Federica and Garner, Matthew

Available at http://clok.uclan.ac.uk/25957/

Hepsomali, Piril, Hadwin, Julie A, Liversedge, Simon Paul ORCID: 0000-00028579-8546, Degno, Federica and Garner, Matthew (2019) The impact of cognitive load on processing efficiency and performance effectiveness in anxiety: evidence from event-related potentials and pupillary responses. Experimental Brain Research, 237 (4). pp. 897-909. ISSN 0014-4819

It is advisable to refer to the publisher's version if you intend to cite from the work. http://dx.doi.org/10.1007/s00221-018-05466-y

For more information about UCLan's research in this area go to http://www.uclan.ac.uk/researchgroups/ and search for <name of research Group>.

For information about Research generally at UCLan please go to http://www.uclan.ac.uk/research/

All outputs in CLoK are protected by Intellectual Property Rights law, including Copyright law. Copyright, IPR and Moral Rights for the works on this site are retained by the individual authors and/or other copyright owners. Terms and conditions for use of this material are defined in the policies page. 
Running Head: EFFICIENCY, EFFECTIVENESS, AND ANXIETY

The Impact of Cognitive Load on Processing Efficiency and Performance Effectiveness in Anxiety: Evidence from Event-Related Potentials and Pupillary Responses

Piril Hepsomali ${ }^{1,3^{*}}$, Julie A. Hadwin ${ }^{1}$, Simon P. Liversedge ${ }^{4}$,

Federica Degno ${ }^{2} \&$ Matthew Garner ${ }^{1,3^{*}}$

${ }^{1}$ Centre for Innovation in Mental Health-Developmental Lab, Department of Psychology, University of Southampton

${ }^{2}$ Centre for Vision and Cognition, Department of Psychology, University of Southampton

${ }^{3}$ Clinical and Experimental Sciences, University of Southampton

${ }^{4}$ School of Psychology, University of Central Lancashire

Author Note

This research was supported by funds from the Economic and Social Research Council

Doctoral Training Centre studentship (Award no:1362797) awarded to Piril Hepsomali, and the School of Psychology, University of Southampton.

Corresponding authors: $\underline{\text { M.Garner@ soton.ac.uk and PHepsomali@ gmail.com }}$ 


\begin{abstract}
Anxiety has been associated with poor attentional control, as reflected in lowered performance on experimental measures of executive attention and inhibitory control. Recent conceptualisations of anxiety propose that individuals who report elevated anxiety symptoms worry about performance and will exert greater cognitive effort to complete tasks well, particularly when cognitive demands are high. Across two experiments we examined the effect of anxiety on task performance and across two load conditions using (1) measures of inhibitory control (behavioural reaction times and eye-movement responses), and (2) task effort with pupillary and electrocortical markers of effort (CNV) and inhibitory control (N2). Experiment 1 used an oculomotor-delayed-response task that manipulated load by increasing delay duration to create a high-load, relative to a low load, condition. Experiment 2 used a Go/No-Go task and load was manipulated by decreasing the No-Go probabilities (i.e., 20\% No-Go in the high load condition and 50\% No-Go in the low load condition). Experiment 1 showed individuals with high (vs. low) anxiety made more antisaccade errors across load conditions, and made more effort during the high load condition, as evidenced by greater frontal CNV and increased pupillary responses. In Experiment 2 individuals with high anxiety showed increased effort (irrespective of cognitive load), as characterised by larger pupillary responses. In addition, N2 amplitudes were sensitive to load only in individuals with low anxiety. Evidence of reduced performance effectiveness and efficiency across electrophysiological, pupillary and oculomotor systems in anxiety provides some support for neurocognitive models of frontocortical attentional dysfunction in anxiety. Keywords: Inhibition, effort, anxiety, pupillary responses, CNV, N2.
\end{abstract}


The Impact of Cognitive Load on Processing Efficiency and Performance Effectiveness in Anxiety: Evidence from Event-Related Potentials and Pupillary Responses

Attentional Control Theory (ACT) proposes that anxiety is characterised by reduced attentional control that impairs processing efficiency and performance effectiveness on goaldirected tasks (Eysenck, Derakshan, Santos, \& Calvo, 2007). It suggests that anxious individuals are motivated to perform tasks to a high standard and to invest additional cognitive resources and effort to achieve performance goals (Berggren \& Derakshan, 2013). Neuropsychological models of anxiety highlight a range of maladaptive biases in top-down attention and cognitive control processes that increase hypervigilance for threat and that negatively impact inhibitory control, cognitive flexibility and working memory (ACT; Eysenck et al., 2007). Anxiety-related deficits in attentional control and executive function have been observed across several behavioural measures including poor performance on the executive attention subtest of the attention network task (ANT; Pacheco-Unguetti, Acosta, Callejas, \& Lupianez, 2010), and slower and less accurate eye-movements in the antisaccade task, particularly when task demand/cognitive load is high (Hepsomali, Hadwin, Liversedge \& Garner, 2017).

The antisaccade task is a simple inhibitory control task that utilises eye movement measures to investigate individual differences in attention control across anxiety and mood disorders. Participants are instructed to look away from a visual cue (i.e. to its mirror location) as quickly and accurately as possible. Introducing a delay period between reflexive saccade inhibition and voluntary saccade generation in the antisaccade task, adds further demands to spatial working memory (Luna \& Velanova, 2011) as evidenced by sustained frontocrotical activation throughout delay periods (Curtis \& D'Esposito, 2003), thus lengthening the delay period results in the persistent and sustained use of frontocortical resources. Consistent with predictions from ACT (Eysenck et al., 2007), anxious individuals 
are more likely to make erroneous eye-movements on antisaccade trials, and are slower (less efficient) at executing antisaccades (Ansari \& Derakshan, 2011b; Garner, Attwood, Baldwin, James, \& Munafo, 2011). Furthermore, anxiety-related deficits in antisaccade performance are greater when processing demands increase (Berggren, Richards, Taylor, \& Derakshan, 2013) (review by Ainsworth \& Garner, 2013).

Slow-wave cortical potentials (SCPs) have also been used to measure associations between anxiety symptoms with effort and performance in cognitive tasks. SCPs are longlasting potentials that sustain from $300 \mathrm{~ms}$ to several seconds and reflect the depolarization (reduced firing threshold) and corresponding increased excitability of cortical neuronal networks. Contingent Negative Variation (CNV; Walter, Cooper, Aldridge, McCallum, \& Winter, 1964) is a SCP that increases with cognitive effort, task difficulty and response preparation and may be sensitive to changes in effort expended by high trait anxious individuals when completing challenging cognitive tasks (Khader, Schicke, Roder, \& Rosler, 2008). To date, electrophysiological correlates of effort in anxiety have not been widely examined. Consistent with models that propose poorer efficiency/greater effort in anxious individuals when task demands are high, one study observed a larger (less positive) CNV at frontal sites during medium (400 ms) and long (1500 ms) delays in a pro/anti-saccade task in individuals who reported increased symptoms of anxiety (Ansari \& Derakshan, 2011a).

Previous studies have similarly shown that larger pupillary responses were associated with resource recruitment and task difficulty (Beatty, 1982; Beatty \& Lucero-Wagoner, 2000; Kahneman \& Beatty, 1966; Karatekin, Marcus, \& Couperus, 2007). For example, high trait anxious individuals elicited larger pupillary responses during tasks that require sustained attention (e.g. simulated driving task; Wilson \& MacLeod, 2003). Pupillary responses have also been linked to anxiety-related deficits in other tasks, such as learning paradigms (e.g. two-bandit arm task; Browning, Behrens, Jocham, O'Reilly, \& Bishop, 2015). Our recent 
study further demonstrated that individuals who reported elevated symptoms of trait anxiety showed enhanced, sustained and inflexible patterns of pupil responding during a high (long delay) (vs. low/short delay) memory load condition and poorer performance (fewer accurate eye-movements) on an anti-saccade task (Hepsomali et al., 2017).

The Go/No-Go task (Donders, 1969) is also an established behavioural measure of inhibitory control (see Simmonds, Pekar, \& Mostofsky, 2008). In this task, participants are typically instructed to respond manually to target stimuli during Go trials and withhold behavioural responses during No-Go trials. Evidence of good attentional and inhibitory control are respectively characterised by few errors of omission (i.e., fewer failures to make a response when one is required) and commission (i.e., an increased tendency to make a response when one is not required) rates. Inhibitory control has been measured via the N2 fronto-central event-related potential (ERP); a negative component at midline frontal sites that appears 250-350 ms after the presentation of a to-be-inhibited stimuli (Eimer, 1993). It is considered to reflect both pre-response inhibition mediated by the ventrolateral prefrontal cortex (VLPFC) and the dorsolateral prefrontal cortex (DLPFC), and conflict monitoring mediated by the anterior cingulate cortex (ACC) (Lavric, Pizzagalli, \& Forstmeier, 2004). In the Go/No-Go task, errors of commission and N2 amplitudes to No-Go stimuli have been found to increase with an increased inhibitory load/effort (i.e. fewer No-Go trials) (Bruin \& Wijers, 2002; Eimer, 1993; Low \& Miller, 1999; Pfefferbaum \& Ford, 1988; Polich, Ellerson, \& Cohen, 1996), and this increase is argued to reflect increased resource required for inhibition.

Further studies suggest that symptoms of psychopathology can modulate N2 amplitudes during response inhibition. For example, N2 amplitudes at frontal sites are reduced in individuals with obsessive-compulsive disorder (Herrmann, Jacob, Unterecker, \& Fallgatter, 2003; Kim, Kim, Yoo, \& Kwon, 2007). Associations between anxiety and the N2 are mixed. 
One correlational study in a non-clinical group of adults observed positive associations between trait anxiety and the N2 amplitude (Righi, Mecacci, \& Viggiano, 2009). Sehlmeyer et al., (2010) also showed a positive association between trait anxiety, the No-Go N2 amplitude and improved behavioural inhibition (reduced false alarms) across unselected individuals reporting mild to moderate anxiety. In contrast, recent evidence suggests that higher levels of anxiety (i.e. in pre-selected groups of trait anxious individuals) were characterised by reduced N2 and hypoactivity in frontal regions (derived from source localisation) during No-Go inhibition trials (Yang \& Li, 2014). This finding is consistent with neuropsychological models of anxiety that highlight frontal hypoactivity and distractibility associated with elevated anxious affect (Bishop, 2009). However, it is important to note that inconsistencies of previous N2 findings make it hard to draw definite conclusions about the N2 and anxiety relationship.

The current paper aimed to extend existing findings to explore the impact of cognitive demand on task performance for individuals with low and high levels of trait anxiety. We utilised convergent measures of electrocortical activity alongside pupil diameter and eyemovement measures to examine associations between anxiety with effort and processing efficiency/ effectiveness. The paper presents two experiments that examined the impact of load on processing efficiency (via electrocortical activity and pupil diameter) and effectiveness (via behavioural performance) in groups of individuals who were screened for high or low levels of trait anxiety symptoms. We extend previous research to use frontal SCPs and N2 amplitudes as novel markers of performance effectiveness in the context of anxiety-related challenges with variation in cognitive load. Experiment 1 considered CNV potentials and pupillary responses during short ( $5 \mathrm{~s}$; low load) and long delay (10 s; high load) periods and measured subsequent eye movement errors and latencies in a pro and antisaccade task. Experiment 2 measured the impact of high and low anxiety symptoms on processing 
efficiency (as reflected in N2 amplitudes and pupillary responses) and performance effectiveness (behavioural responses) during No-Go and Go trials under low (50\% Go, \%50 No-Go) and high (80\% Go, \%20 No-Go) load conditions.

We anticipated that individuals with elevated levels of trait anxiety would show impaired task performance, as reflected in fewer and slower accurate eye-movements on antisaccade trials and lowered processing efficiency, as indicated by greater midline-frontal potentials (larger CNVs) and larger pupillary responses, in the context of increased cognitive load (Experiment 1). In addition, we expected that individuals with elevated levels of trait anxiety would show less inhibitory control (more No-Go errors) and increased effort (i.e., larger pupillary responses and reduced N2 amplitudes) during inhibitory control and in the context of increased cognitive load (i.e., more Go trials relative to No-Go trials; Experiment 2).

\section{General Method}

\section{Participants}

Fifteen high trait anxious and 15 low trait anxious participants were selected to take part in the study from a university participant pool based on scores on the trait version of the State-Trait Anxiety Inventory (Spielberger, Gorusch, Lushene, Vagg, \& Jacobs, 1983). Consistent with previous research (Ansari, Derakshan, \& Richards, 2008; Hepsomali, Hadwin, Liversedge, \& Garner, 2017), participants who scored $\leq 35$ were categorised as low anxious $(\mathrm{LA} ; n=15$, mean STAI-T $=28.80, S . D .=2.93$, in $=23$, max $=33$, mean age $=$ $20.27 ; 10$ females) and those scoring $\geq 50$ as high anxious (HA; $n=15$, mean STAI-T $=$ 59.93, S.D. $=7.93, \min =50, \max =77 ;$ mean age $=20.60 ; 13$ females $)$. Levels of state anxiety (STAI-S) prior to the task were $M=44.80$, S.D. $=7.94$ for high trait anxious and $M=$ 26.60, S.D. $=5.52$ for low trait anxious individuals. 
All participants had normal or corrected-to-normal vision and were right-handed (as verified by the Edinburgh Handedness Inventory; Oldfield, 1971). Participants confirmed that they had not taken drugs, alcohol, or medication on the day preceding testing. They received either course credits or $£ 10$ for participation. All participants provided informed consent. The research protocol was approved by the University's Ethics and Research Governance committees.

\section{Data Acquisition}

Electroencephalographic (EEG) data were recorded from $64 \mathrm{Ag} / \mathrm{AgCl}$ scalp electrodes mounted in an elastic electrode cap (EasyCap GmbH, Germany) and Synamps RT amplifier (Neuroscan, Inc). Electrode sites were determined using the international 10-20 system. All electrodes were referenced online to the nose and later re-referenced offline to the average of all electrodes. AFz was used as a ground electrode. Horizontal eye movements (HEOG) were recorded by placing electrodes laterally on the outer canthi of the eyes and vertical eye movements (VEOG) were recorded from electrodes placed below and above the left eye. Impedances were kept below $5 \mathrm{k} \Omega$ for all channels. Signals were amplified in a DC mode at $100 \mathrm{~Hz}$ and sampled at $1000 \mathrm{~Hz}$ using Neuroscan 4.5 (Neuroscan, Inc).

Eye movements and pupillary responses were recorded using SR EyeLink 1000 desktop-mounted eye tracking system (SR Research, ON, Canada) with a sampling rate of 1 kHz. Pupillary responses were recorded using a Centroid model based on pupil diameter in millimetres. The presentation was controlled by Experiment Builder v1.10.1025 software (SR Research Ltd., Ontario, Canada) on a 19-inch ViewSonic (P227f) monitor.

\section{Experimental Tasks and Procedure}

All stimuli were presented on a grey background $(\mathrm{R}=117, \mathrm{G}=116, \mathrm{~B}=116)$, and all trial screen displays had a mean luminosity of 116. Participants were calibrated (3-point display) before completing 10 practice trials for each experiment and the experimental tasks. 
Participants were instructed to maintain central fixation and minimise movements.

Participants could take small breaks and were re-calibrated where appropriate. Participants were seated in front of the computer and eye-tracking system with their chin and head supported at a viewing distance of $70 \mathrm{~cm}$. Experiments were presented in random order.

\section{Experiment 1}

\section{Antisaccade Task}

Participants initially completed a successful drift correction. As seen in Figure 1, a central cue was then presented for $1000 \mathrm{~ms}$ (letters $\mathrm{O}$ or $\mathrm{X}$ ) and was used to indicate the trial type (respectively prosaccade or antisaccade). After a short (5 seconds) or long (10 seconds) delay, a grey ellipse-shaped target $\left(\mathrm{R}=79, \mathrm{G}=78, \mathrm{~B}=78 ; 3.3^{\circ} \times 6^{\circ}\right)$ was displayed for $1500 \mathrm{~ms}$ at $11^{\circ}$ either to the left or right side of the central fixation cross. Participants were instructed to look towards the target (prosaccade) or away from the target (antisaccade). The task consisted of 200 trials (100 long and 100 short delay) presented in a random order across 2 blocks of 100 trials (each comprising 50 prosaccade trials and 50 antisaccade trials).

\section{Data Pre-Processing}

Saccades. Saccade accuracies and latencies (following target onset) were extracted for the first valid saccade on each trial (i.e. latencies $>80 \mathrm{~ms}$, velocities $>30 \%$, and amplitudes $>3^{\circ}$ ) (Ansari \& Derakshan, 2010). An erroneous saccade was defined as the first saccade landing outside the relevant interest area (i.e. the grey ellipse-shaped target area on prosaccade trials, or the mirror location on antisaccade trials).

Pupillary responses. Pupillary responses during the delay period (5000 ms and 10000 ms) were pre-processed following standard procedures (cf. Beatty \& Lucero-Wagoner, 2000; Bradley, Miccoli, Escrig, \& Lang, 2008; Granholm, Morris, Asarnow, Chock, \& Jeste, 2000): epoching, blink-artefact removal and linear interpolation, averaging, baseline correction and selection of the peak pupil diameter by using MATLAB R2013a (The Math-Work, Inc., MA, 
USA). Peak pupillary responses (maximum pupil diameter) for correct trials were calculated during the $5000 \mathrm{~ms}$ delay period (A), and during the first (B) and second (C) $5000 \mathrm{~ms}$ of the 10000 ms delay period. Participants were not aware if the current trial was a short delay or a long delay trial until the end of 5 seconds, therefore responses during the short delay $(A+B / 2)$ were compared against responses during the long delay (C). Artefacts and blinks were defined as very short increases or decreases of at least $0.375 \mathrm{~mm}$ within $20 \mathrm{~ms}$ (Partala \& Surakka, 2003) and were removed. Missing values were corrected by linear interpolation (\% interpolated $=10.28)$.

Event-Related Potentials. EEG recordings were band pass filtered $(0.1-30 \mathrm{~Hz})$, corrected for blink/eye-movement artefacts via VEOG (minimum sweeps:20, duration: $400 \mathrm{~ms})$, re-referenced and epoched from $100 \mathrm{~ms}$ pre-delay and $1000 \mathrm{~ms}$ post-delay, resulting in -100 to $6100 \mathrm{~ms}$ and -100 to $11100 \mathrm{~ms}$ epochs for short and long delays, respectively. All epochs were re-referenced to the average of all electrodes, and baseline corrected (100ms preceding the stimulus onset). Consistent with previous studies we excluded trials with erroneous saccadic responses (Ansari \& Derakshan, 2011a). Grand average waveforms were computed separately for short and long delay trials. SCPs were calculated during the 5000 ms delay period (A), and during the first (B) and second (C) $5000 \mathrm{~ms}$ of the $10000 \mathrm{~ms}$ delay period. Responses during short delay $(\mathrm{A}+\mathrm{B} / 2)$ were compared against responses during the long delay (C). Large positive and negative evoked potentials within $700 \mathrm{~ms}$ after stimulus offset (i.e. prior to SCP) were removed from the calculation of average SCP amplitudes (i.e. average amplitudes were calculated from 700-5000 ms). Consistent with (Studer et al., 2014) we also included a supplementary analysis of mean preparatory CNV activity between -400 ms and $-100 \mathrm{~ms}$ before target presentation and consistent with (Ansari \& Derakshan, 2011b) we examined mean activity immediately prior to target onset $(-50 \mathrm{~ms}$ before to $50 \mathrm{~ms}$ after 
target presentation, i.e. before eye movement execution). Both SCP and CNV analyses are time-locked to pre-response (i.e. eye movements) period.

\section{Results}

\section{Saccade Error and Latency.}

Saccade errors and latencies were analysed in two separate $2 \times 2 \times 2$ repeated measures ANOVAs with Group (low-anxious, high-anxious) as the between subject factor and Saccade Type (anti-, prosaccade) and Delay (short, long) as the within subject factors. Participants made more errors on antisaccade $(M=46.81, S . E .=2.92)$ vs. prosaccade trials $(M=10.86$, S.E. $=0.91),\left[F(1,28)=149.39, p=.001, \eta^{2}=.84\right]$, particularly following the short delay [Saccade Type x Delay interaction, $F(1,28)=9.12, p<.01, \eta^{2}=.24$; Antisaccade $\mathrm{M}_{\text {short }}=50.2$, S.E. $=2.6, \mathrm{M}_{\mathrm{long}}=43.4$, S.E. $=3.6 ;$ Prosaccade $\mathrm{M}_{\text {short }}=10.9$, S.E. $=0.9, \mathrm{M}_{\mathrm{long}}=10.8$, S.E. $=1.3]$. A significant Group x Saccade Type interaction $\left[F(1,28)=4.66, p=.04, \eta^{2}=.14\right]$ was characterised by a greater number of eye-movement errors in high vs. low anxious individuals on antisaccade trials only, $t(28)=2.28, p=.03$, Antisaccade Mнн $=53.20$, S.E. $=$ 4.13, $\mathrm{M}_{\mathrm{LA}}=40.13$, S.E. $=4.13 ;$ Prosaccade $\mathrm{M}_{\mathrm{HA}}=11.20$, S.E. $=1.30, \mathrm{M}_{\mathrm{LA}}=10.53$, S.E. $=1.30$ (see Figure 2).

A corresponding analysis of eye-movement latencies revealed that participants were slower to make correct saccades on antisaccade $(M=366.99$, S.E. $=12.52)$ vs. prosaccade trials $(M=275.78$, S.E. $=11.05),\left[F(1,28)=83.77, p<.01, \eta^{2}=.74\right]$, and following short $(M=$ 330.61$, S.E. $=13.43)$ compared to long delay trials $(M=312.16$, S.E. $=9.32),[F(1,28)=4.79, p$ $\left.=.03, \eta^{2}=.14\right]$. All other main and interaction effects were not significant $(F \mathrm{~s}<1 p s>.1)$.

\section{Pupillary Responses}

Amplitude and latency of peak pupil responses were entered into separate 2 x 2 repeated measures ANOVA with Group (low-anxious, high-anxious) as the between subject factor and Delay (short, long) as the within subject factor. Greenhouse-Geisser corrections 
were applied where sphericity was violated. Follow-up supplementary time-series analyses of pupil diameter examined effects of delay and anxiety over-time across 250 consecutive 20 ms epochs as per previous research (Geva, Zivan, Warsha, \& Olchik, 2013; Hepsomali et al., 2017). The results also showed a Group x Delay interaction $\left[F(1,28)=4.59, p=.04, \eta^{2}=.16\right]$ High (vs low) anxious individuals had increased peak pupil amplitude during long delays only, $t(28)=2.15 p=.04$. In addition, high anxious individuals had increased peak pupil amplitude during long compared to short delays, $t(14)=2.48 p=.02$ [Long Delay $\mathrm{M}_{\mathrm{HA}}=0.21$, S.E. $=0.02, \mathrm{M}_{\mathrm{LA}}=0.132$, S.E. $=0.02$; Short Delay $\mathrm{M}_{\mathrm{HA}}=0.16 .$, S.E. $=0.01, \mathrm{M}_{\mathrm{LA}}=0.15$, S.E. $=0.01]$ (see Figure 2). Comparable analyses of peak latency did not reveal effects of group, delay, or their interaction $(F s<1 p s>.1)$.

\section{Contingent Negative Variation}

Statistical comparisons of ERP activity were conducted on data from three midline electrodes $(\mathrm{Fz}, \mathrm{Cz}, \mathrm{Pz})$ (based on previous findings showing no laterality in delay-related slow shifts and in effort-related slow-waves; see Ansari \& Derakshan, 2011a; Rämä, Carlson, Kekomi, \& Hämäläinen, 1995). Mean amplitudes of the CNV were analysed with a 2 x 3 x 2 repeated measures ANOVA with Group (low-anxious, high-anxious) as the between subject factor and Area (frontal, central, parietal) and Delay (short, long) as the within subject factors. Where assumptions of sphericity were violated, Greenhouse-Geisser corrections were applied. The results showed a Group x Delay interaction $\left[F(1,28)=4.82, p=.03, \eta^{2}=.18\right]$ that was characterised by greater CNV during the long delay only in high anxious individuals compared to low anxious individuals $t(28)=1.40, p=.04$, [Long Delay MнA $=0.04$, S.E. $=$ $0.68, \mathrm{M}_{\mathrm{LA}}=0.90$, S.E. $=0.68 ;$ Short Delay $\mathrm{M}_{\mathrm{HA}}=0.38 .$, S.E. $=0.54, \mathrm{M}_{\mathrm{LA}}=0.52$, S.E. $\left.=0.44\right]$. There were no other main or interaction effects $(\mathrm{Fs}<1 \mathrm{ps}>.1)$.

We also explored two additional time windows between $-400 \mathrm{~ms}$ and $-100 \mathrm{~ms}$ and between $-50 \mathrm{~ms}$ to $+50 \mathrm{~ms}$. A corresponding $2 \times 3 \times 2$ repeated measures ANOVA of mean 
CNV amplitudes (-400 to -100 ms before target onset) provided evidence of a Group x Delay $\mathrm{x}$ Area interaction $\left[F(1,58)=3.05, p=.05, \eta^{2}=.11\right]$ characterised by greater $\mathrm{CNV}$ in high vs. low anxious individuals during the long delay at frontal sites $t(14) 2.23, p=.04$, [Long Delay $\mathrm{M}_{\mathrm{HA}}=-0.27$, S.E. $=0.91, \mathrm{M}_{\mathrm{LA}}=1.94$, S.E. $=0.94 ;$ Short Delay $\mathrm{M}_{\mathrm{HA}}=1.11 .$, S.E. $=0.70$, $\mathrm{M}_{\mathrm{LA}}=1.54$, S.E. $=0.73$ ] (see Figure 2). A corresponding analysis of CNV amplitudes $-50 \mathrm{~ms}$ to $+50 \mathrm{~ms}$ showed no main or interaction effects (Fs $<1 p s>.1)$.

\section{Associations between Measures of Effort/Processing Efficiency and Processing effectiveness}

We examined the association between the two indices of effort; peak pupillary responses and mean CNV amplitudes using Pearson product-moment correlations. There was a significant negative correlation between CNV amplitude at frontal site and pupillary responses in antisaccade trials during long delay trials $(r=-.48, p=.01)$, indicating that the two indices of effort were associated (Figure 3). The associations between behavioural (saccade error rate and saccade onset latency) and physiological (CNV and pupillary responses) also showed a significant positive correlation between error rates of antisaccades during short delay trials and pupillary responses during short delay trials $(r=.37, p=.04)$. All other correlations were not significant $(p s>.1)$.

\section{Discussion- Experiment 1}

Experiment 1 examined processing efficiency (via electrophysiological and pupillometric measures) and effectiveness (using saccadic measures) in high and low anxious adults using a pro- and antisaccade task with high and low cognitive load conditions. Considering processing efficiency and anxiety, the results showed that high (vs. low) anxious individuals produced greater $\mathrm{CNV}$ and larger pupillary responses during long delay trials. In addition, anxiety also impacted performance effectiveness, with adults in the high (vs. low) anxiety group making more eye movement errors on antisaccade trials. 
Evidence of increased effort as reflected in greater CNV and increased pupillary responses in high-anxious individuals, particularly under experimental tasks with increased cognitive load, is consistent with models that predict lowered processing efficiency in anxiety when task demands are high (Berggren et al., 2013; Mandrick, Peysakhovich, Rémy, Lepron, \& Causse, 2016). Our findings extend evidence of greater preparatory CNV in anxiety (Ansari \& Derakshan, 2011a) and reveal sustained preparatory potentials over a longer time period (5-10 seconds) prior to response. Our supplementary analysis of cortical potentials prior to target onset (-400 to $-100 \mathrm{~ms}$ ) suggested that the effect of anxiety was strongest at frontal sites. This finding is consistent with previous research (Ansari \& Derakshan, 2011a) and evidence from neuroimaging that implicates frontal regions in antisaccade performance accuracy (Ford, Goltz, Brown, \& Everling, 2005). Though the predicted greater CNV in anxiety was observed, a large sustained negative potential that characterises 'classic' CNV profile was not found. The findings are, however, consistent with previous studies that have reported less positive (vs. large negative) CNVs in demanding tasks that require participants to wait for long periods after the instructional cue and before executing the response to target (Turner et al., 2015).

Links between $\mathrm{CNV}$ with the pupillometry findings provide convergent evidence of increased effortful processing in anxiety. Larger peak pupillary responses were observed in high anxious individuals during long delay trials and in long versus short delays, while low anxious individuals exhibited similarly small pupillary responses during both delay conditions. Previous research has similarly shown that high trait anxious individuals exhibit enhanced, sustained and inflexible patterns of pupil responding during affective stimulus processing and delay periods that precede errors in pro/antisaccade performance (Hepsomali et al., 2017). Here too we found evidence of increased antisaccade errors in high anxious individuals due to the inefficiency of inhibitory control, consistent with reduced performance 
effectiveness (Eysenck et al., 2007). However, anxiety did not modulate eye-movement latency nor interact with delay.

The results also showed that antisaccade performance was more accurate and faster following the long (rather than short) delay. In the current paradigm, the likelihood a target appeared at $5 \mathrm{~s}$ was 50\%, whereas the likelihood of the target appearing at $10 \mathrm{~s}$ (after the nonappearance of the target at $5 \mathrm{~s}$ ) was $100 \%$. Consequently, on long delay trials participants were able to prepare accurate and quick responses with confidence (from 5 seconds onwards). In support, pupillometry data provides evidence that participants were sensitive to temporal characteristics of the trial and re-engaged resources after the non-appearance of the target at 5 s on long $10 \mathrm{~s}$ trials (see Figure 4). In support of this interpretation, performance in the short delay was more aligned to effort (pupillary) indices, indicating that the demonstration of associations between effort and performance may be more evident across shorter time frames.

In Experiment 2 we aimed to provide further evidence for the effect of cognitive load in anxiety on processing effectiveness and efficiency. We used high and low load versions of a Go/No-Go task to generate a behavioural index of inhibitory control (using behavioural reaction times) and reliable indices of effort (i.e., N2 amplitudes and pupillary responses). Load was increased by decreasing the frequency of No-Go trials.

\section{Experiment 2}

\section{Go/No-Go Task}

As seen in Figure 5, after a successful drift correction, a central fixation (1000 ms) and a central cue (letters $\mathrm{O}$ or $\mathrm{X}$ ) was presented for $1000 \mathrm{~ms}$ to indicate the type of the trial (respectively Go or No-Go). In Go trials participants were asked to press a response button as soon as they saw the Go cue (the letter O) but withhold their responses if they saw the NoGo cue (the letter X). The task consisted of 600 trials presented across 6 blocks of 100 trials (trial and block order were randomised). There were 3 blocks that required increased 
cognitive load/ effort (20\% No-Go, $80 \%$ Go trials) and 3 low load/effort blocks (50\% No-Go, $50 \%$ Go trials).

\section{Data Pre-processing}

Behavioural performance. We computed mean reaction times (RTs) for Hits in high load ( $80 \%$ Go) and low load (50\% Go) trials, and percentage of commission errors (i.e., false alarms; not inhibiting when required) in high load (20\% No-Go) and low load (50\% No-Go) trials. Mean RTs were not calculated for the small percentage of commission errors $(1.36 \%)$. Omission errors (i.e., misses; not responding when required) $=2.05 \%$.

Pupillary responses. Pupillary responses for the Go/No-Go period (1000 ms) were pre-processed using previous standard procedures (cf. Beatty \& Lucero-Wagoner, 2000; Bradley et al., 2008; Granholm et al., 2000) using MATLAB R2013a (The Math-Work, Inc., MA, USA). Firstly, blinks and artefacts (very short increases or decreases of at least 0.375 mm within 20 ms; (Partala \& Surakka, 2003) were removed. Missing values were corrected by linear interpolation (9.89\% interpolated) and a baseline correction (relative to the start of epoch) was applied. Peak pupillary responses (maximum pupil diameter) and latency to peak (ms) were calculated during the $1000 \mathrm{~ms}$ Go/No-Go period. Distributions of amplitude and latency of peak pupillary responses were examined and met assumptions of normality using Shapiro-Wilk's test $(p s>.05)$.

Event-related potentials. The continuous EEG recordings were band pass filtered offline with a high-pass cut-off of $0.1 \mathrm{~Hz}$ and a low-pass cut-off of $30 \mathrm{~Hz}$, using a zero-phase shift FIR (Finite Impulse Response) filter. Eye blinks in the continuous EEG data were identified and corrected (via VEOG, minimum sweeps:20, duration: 400ms). Muscle bursts were manually selected and removed from further analyses. The data were epoched $100 \mathrm{~ms}$ pre-cue and $500 \mathrm{~ms}$ post-cue resulting in - 100 to $2600 \mathrm{~ms}$ epochs. All epochs were rereferenced to the average of all electrodes, and baseline corrected $(100 \mathrm{~ms}$ preceding the 
stimulus onset). Consistent with previous studies (Sehlmeyer et al., 2010), trials with erroneous responses (errors of commission and omission) were excluded. Grand average waveforms were computed separately for $80 \%$ Go, $20 \%$ No-Go, $50 \%$ Go, $50 \%$ No-Go trials. Statistical comparisons of ERP activity were conducted on the N2 (200-350 ms) component from three midline electrodes $(\mathrm{Fz}, \mathrm{Cz}, \mathrm{Pz})$ on the basis of previous Go/No-Go studies (Bruin \& Wijers, 2002; Falkenstein, Hoormann, \& Hohnsbein, 1999; Righi, Mecacci, \& Viggiano, 2009; Sehlmeyer et al., 2010). Distributions of the N2 amplitude was examined and met assumptions of normality (Shapiro-Wilk's test, p's > .05).

\section{Results}

\section{Go/ No-Go Performance}

Reaction times of hits and percentage of commission errors were entered into separate 2 x 2 mixed design ANOVA with Group (low-anxious, high-anxious) as the between subject factor and Inhibitory Load (High, Low) as the within subject factor. Greenhouse-Geisser corrections were applied where assumptions of sphericity were violated. Participants made faster Go responses (GoRT) and more commission errors (CE) on No-Go trials in high load compared to low load blocks $\left[G o R T_{H i g h}=366.81 \mathrm{~ms}\right.$ S.E. $=9.95, G o R T_{\text {Low }}=387.58 \mathrm{~ms}$ S.E. $=$ 7.83, $F(1,28)=13.76, p=.001, \eta^{2}=.33 ; C E_{\text {High }}=2.33 \%$ S.E.= $.27, C E_{\text {Low }}=0.40 \%$ S.E. $=.08$, $\left.F(1,28)=56.71, p=.001, \eta^{2}=.66\right)$. There were no other significant main or interaction effects $(F \mathrm{~s}<1 p s>.1)$.

\section{Pupillary Responses}

Amplitude and latency of peak pupil dilation (i.e. maximum pupil diameter) were entered into separate $2 \times 2 \times 2$ repeated measures of ANOVA with Group (low-anxious, highanxious) as the between subject factor and Trial (Go, No-Go) and Inhibitory Load (High, Low) as within subject factors. Follow-up supplementary time-series analyses of pupil 
diameter examined effects of delay and anxiety over time across 50 consecutive $20 \mathrm{~ms}$ epochs as per previous research (Geva et al., 2013; Hepsomali et al., 2017).

Peak dilation responses were larger during high load blocks $(M=0.05 \mathrm{~mm}$, S.E. $=$ 0.006) compared to low load $(M=0.03 \mathrm{~mm}$, S.E. $=0.005)$ blocks, $F(1,28)=8.22, p=.001, \eta^{2}$ $=.22$. A Trial $\mathrm{x}$ Inhibitory Load interaction $\left[F(1,28)=7.14, p=.01, \eta^{2}=.20\right]$ was characterised by increased peak pupillary responses to No-Go stimuli during high load compared to No-Go low load trials, $t(29)=3.55, p=.001$, High Load $\mathrm{M}_{\mathrm{No}-\mathrm{Go}}=0.06$, S.E. $=$ 0.009, $\mathrm{M}_{\mathrm{Go}}=0.04$, S.E. $=0.006 ;$ Low Load $\mathrm{M}_{\mathrm{No}-\mathrm{Go}}=0.03 .$, S.E. $=0.005, \mathrm{M}_{\mathrm{Go}}=0.04$, S.E. $=0.006$. Supplementary time series analyses suggest that differences in pupil dilation between high and low load conditions in No-Go trials were most pronounced from 200-400 ms after the No-Go cue onset (see Figure 6). The time-series analyses also provided evidence that high anxious individuals exhibited an increase in pupil diameter from 240-340 ms compared to low anxious individuals (irrespective of trial and load, see Figure 6).

Comparable analyses of peak dilation latency did not reveal significant effects of group, trial, inhibitory load or their interaction $(F \mathrm{~s}<1 p s>.1)$.

\section{N2 Amplitudes}

N2 amplitudes were entered into a $2 \times 3 \times 2 \times 2$ mixed design ANOVA with Group (low-anxious, high-anxious) as the between subject factor and Area (frontal, central, parietal), Trial (Go, No-Go) and Inhibitory Load (High, Low) as the within subject factors. N2 amplitudes were larger on No-Go $(M=-1.98 \mu \mathrm{V}, S . E .=0.23)$ compared to Go trials $(M=-$ $1.54 \mu \mathrm{V}$, S.E. $\left.=0.20), F(1,28)=8.54, p=.001, \eta^{2}=.26\right)$, and at frontal $(M=-4.01 \mu \mathrm{V}$, S.E. $=$ $0.44)$ and central $(M=-1.04 \mu \mathrm{V}$, S.E. $=0.32)$ compared to parietal sites $(M=-0.23 \mu \mathrm{V}$, S.E. $=$ $\left.0.28), F(1,28)=30.80, p=.001, \eta^{2}=.56\right)$. A Trial $x$ Inhibitory Load interaction $[F(1,28)=7.90$, $\left.p=.01, \eta^{2}=.24\right]$ was characterised by larger N2 amplitudes under high load for No-Go compared to Go trials $(t(28)=4.56, p=.001)$. N2 amplitudes were similar across No-Go and 
Go trials under low load $(t(28)=.51, p=.60)$, High Load $\mathrm{M}_{\mathrm{No}-\mathrm{Go}}=-2.04$, S.E. $=0.22, \mathrm{M}_{\mathrm{Go}}=-$ 1.23, S.E. $=0.17 ;$ Low Load $\mathrm{M}_{\mathrm{No}-\mathrm{Go}}=-1.12 .$, S.E. $=0.26, \mathrm{M}_{\mathrm{Go}}=-1.85$, S.E. $=0.29$ (see Figure 7). A Trial $\mathrm{x}$ Area interaction $\left[F(1,28)=16.12, p=.001, \eta^{2}=.40\right]$ was characterised by larger $\mathrm{N} 2$ at frontal sites for No-Go relative to Go trials $(t(28)=6.85, p=.001)$, but not at central $(t(28)=.99, p=.32)$, and parietal $(t(28)=1.81, p=.08)$ sites $\left[\mathrm{Fz} \mathrm{M}_{\mathrm{No}-\mathrm{Go}}=-4.73\right.$, S.E. $=0.50$, $\mathrm{M}_{\mathrm{Go}}=-3.28, S . E .=0.41 ; \mathrm{Cz}_{\mathrm{No}-\mathrm{Go}}=-1.92 .$, S.E. $=0.41, \mathrm{M}_{\mathrm{Go}}=-0.96$, S.E. $=0.28 ; \mathrm{Pz}_{\mathrm{No}-}$ $\left.\mathrm{Go}=-0.09 ., S . E .=0.28, \mathrm{M}_{\mathrm{Go}}=-0.37, S . E .=0.32\right]($ See Figure 7$)$.

These effects were subsumed under a four-way Group x Trial x Inhibitory Load x Area interaction $\left[F(1,56)=3.96, p=.02, \eta^{2}=.14\right]$. Follow-up ANOVAs were conducted at each site separately. At the frontal site there was evidence of a Group x Trial x Inhibitory Load interaction $\left[F(1,27)=4.57, p=.04, \eta^{2}=.15\right]$, but no effects were observed at central or parietal sites. For the low anxiety group frontal N2 differed across Trial x Load $[F(1,14)=$ $\left.18.55, p=.001, \eta^{2}=.58\right]$ and was characterised by larger No-Go N2 amplitudes under high (vs. low) inhibitory load $(t(14)=2.67, p=.01)$. In contrast for the high anxiety group, No-Go N2 amplitudes did not differ across high and low load [High Load MнA= -4.49, S.E. $=0.75$, $\mathrm{M}_{\mathrm{LA}}=-5.06, S . E .=0.75 ;$ Low Load $\left.\mathrm{M}_{\mathrm{HA}}=-3.25, S . E .=0.51, \mathrm{M}_{\mathrm{LA}}==3.86, S . E .=0.76\right]$. (see Figure 8). NoGo-N2 amplitudes did not differ between groups under high load $t(28)=1.15$, p $=.25$, or low load $\mathrm{t}(28)=0.8, \mathrm{p}=.93$ No other effects were significant $(\mathrm{Fs}<1 \mathrm{ps}>.1)$.

\section{Associations between Behavioural and Physiological Measures}

We examined the associations between behavioural (RTs of hits and percentage of commission errors) and physiological (N2 and pupillary responses indices) using Pearson product-moment correlations. There was a significant positive correlation between RT of hits under high load and N2 activity at Fz during go trials under high load $(r=.52, p=.004)$. Also, the proportion of commission errors under high load was negatively correlated with N2 
activity at Fz during No-Go trials under high load $(r=-.38, p=.03)$. All other correlations were not significant $(p s>.1)$.

\section{Discussion- Experiment 2}

Experiment 2 examined behavioural, electrophysiological and pupillometric correlates of inhibitory control in high and low anxious groups under high and low inhibitory load/effort in a Go/No-Go task. Across groups, participants produced faster, but less accurate behavioural responses and larger pupillary responses, in high (vs. low) load blocks, especially during inhibitory control (No-Go trials). Pupillary responses were larger for individuals in the high (vs. low) anxiety group, indicating increased effort across the two experimental conditions. In addition, individuals in the low anxiety group produced larger N2 amplitudes in high (vs. low load) load blocks in the frontal region during inhibitory control (No-Go trials). As larger pupillary responses and reduced N2 amplitudes were associated with, enhanced effort and impaired inhibitory control, respectively, the results indicate that high anxious individuals had impaired inhibitory control, although they exerted more overall cognitive effort compared to low anxious individuals.

Consistent with previous studies in unselected samples (Bruin \& Wijers, 2002; Low \& Miller, 1999), the behavioural data showed a reduction in reaction time for Go trials and an increase in error rate for No-Go during high load blocks, indicating poorer monitoring and lower inhibitory control. An effect of anxiety on reaction times (on Go trials) or error rates (on No-Go trials) was not observed (Righi et al., 2009; Sehlmeyer et al., 2010; Yang \& Li, 2014). While we anticipated some association between cognitive load and anxiety, the results raise the possibility that the Go/No-Go task (with Go/No-Go ratios of 80:20 and 50:50) might not place sufficiently high cognitive demands on the executive functions to be sensitive to behavioural differences in individuals with elevated levels of anxiety. Another explanation for the lack of behavioural differences might be that the Go/No-Go task might measure the 
ability to react to infrequent conflict (No-Go trials) rather than sustained efficiency of inhibitory control.

In accordance with previous research, N2 component amplitudes were moderated by trait anxiety (Herrmann, Jacob, Unterecker, \& Fallgatter, 2003; Kim, Kim, Yoo, \& Kwon, 2007; Righi et al., 2009; Sehlmeyer et al., 2010). Specifically, the results showed that the N2 amplitude was linked to response monitoring (via its positive association with increased RTs on Go trials in the high load condition) and with error detection (via its negative correlation with errors on No-Go trials in the high load condition), with this latter finding indicating that it was preceded behavioural inhibition in this task. The current study found a greater frontal No-Go N2 in high load vs. low load in low anxious individuals, whereas high anxious individuals No-Go N2 amplitudes were small across both load conditions. These findings complement evidence of reduced N2 amplitudes at frontal areas in patients with obsessivecompulsive disorder in inhibitory control tasks (Herrmann et al., 2003; Kim et al., 2007); (Righi et al., 2009; Sehlmeyer et al., 2010). Smaller No-Go N2 amplitudes at frontal sites might reflect reduced recruitment of frontal cortex (Bishop, 2009) in high anxious individuals, especially during cognitively demanding high load blocks in which low anxious exhibit enhanced frontal No-Go N2 amplitudes. Indeed, low anxious individuals' capacity to reduce inhibitory control/effort in low load trials is consistent with flexible resource allocation (pupillary responding) in low anxious individuals in other tasks (e.g. load-dependent pupillary responses in low anxious individuals (Browning, Behrens, Jocham, O'Reilly, \& Bishop, 2015).

Evidence of larger and more sustained pupillary responses in high (vs. low) anxious group supports the finding in Experiment 1. In the current study anxiety group differences were most pronounced between $240-340 \mathrm{~ms}$ after the onset of the cue (both Go and No-Go) and were slower to return to baseline. These findings are also consistent with findings from 
previous research which suggests that anxious individuals invest greater effort to achieve performance goals (Hepsomali et al., 2017). In contrast to the findings in Experiment 1, the findings in this second study did not reveal condition specific increased pupillary responses in high (vs. low) anxious individuals, i.e., during high load blocks or in experimental trials that required inhibitory control. Consistent with previous findings the results indicated sustained but inflexible pupillary responses in anxiety (Hepsomali et al., 2017), indicating that individuals who report elevated anxiety symptoms showed increased cognitive effort regardless of task demand and type.

\section{General Discussion}

Across two experiments we provide evidence of reduced performance effectiveness and efficiency across electrophysiological, pupillary and oculomotor systems to support neuropsychological models of anxiety that highlight broad deficits as a 'hidden cost' of anxiety (Ansari \& Derakshan, 2011a; Eysenck et al., 2007). Taken together, our findings provide evidence of impaired inhibitory control and of compensatory strategies (i.e., increased cognitive effort) in anxiety (Eysenck et al., 2007). These effects may be attributable to dysfunctional prefrontal recruitment during inhibitory control (Bishop, 2009) and response preparation. The results further suggest that anxiety is characterised by dysfunction in the early stages of processing associated with response/conflict monitoring and error detection, but not in the late stages that involves the evaluation of inhibition and performance of inhibitory control.

According to attentional control theory (Eysenck et al., 2007), anxiety increases bottom-up and decreases top-down processing, resulting in less efficient and effective inhibitory control. Although behavioural responses (commission errors/false alarms) showed comparable response effectiveness in high and low anxious individuals (Exp 2), we did observe greater eye-movement error rates on antisaccade trials in high (vs. low) anxious 
individuals, consistent with evidence of oculomotor control deficits in anxiety (Exp1) (see Ainsworth \& Garner, 2013, for a review).

Current models of anxiety postulate that high (vs. low) anxious individuals have impaired inhibitory control (Eysenck et al., 2007) and reduced DLPFC control of attention (Bishop, 2009). However, previous research has shown that individuals who report elevated anxiety exert greater cognitive effort and allocate more cognitive resources in order to compensate for task performance and maintain performance effectiveness and efficiency particularly under high load conditions (Eysenck \& Derakshan, 2011; Eysenck et al., 2007). It is possible that a curvilinear relationship exists between trait anxiety and inhibitory control in which moderate anxiety increases behavioural and cortical inhibition, possibly through increased levels of compensatory effort, arousal and state anxiety in low load tasks, but where higher levels of trait anxiety reduce behavioural and cortical inhibition, especially when compensatory processing is limited by greater task demands.

The behavioural results of the Go/No-Go task suggest comparable response inefficiency in high and low anxious individuals. That is, we did not observe the effects of effort/load on behavioural (RTs of hits) and oculomotor (saccade onset latencies) indices of performance, rather we observed effects on electrophysiological and pupillary measures (e.g., Righi et al., 2009; Roche, Garavan, Foxe, \& O'Mara, 2005). In addition, group effects on task performance were most evident in Experiment 1, perhaps advocating the use of delay manipulations in oculomotor tasks to examine individual differences in effortful processing in anxiety.

\section{Limitations and Future Research}

In the current study participants were selected from a participant pool according to their self-reported trait anxiety scores. Although previous studies have shown similar effect sizes in clinical and subclinical groups for threat-related attentional bias (Bar-Haim, Lamy, 
Pergamin, Bakermans-Kranenburg, \& van, 2007) and attentional control (Najmi, Amir, Frosio, \& Ayers, 2015; Vytal, Cornwell, Letkiewicz, Arkin, \& Grillon, 2013) findings require replication in clinical groups. Our findings support frameworks that highlight working memory and executive control processes as therapeutic targets in anxiety and invite future trial protocols to examine improvements in processing efficiency and effectiveness across behaviour, peripheral and electrocortical markers, in addition to self-report symptoms of anxiety. 


\section{References}

Ainsworth, B., \& Garner, M. (2013). Attention control in mood and anxiety disorders: evidence from the antisaccade task. Hum Psychopharmacol, 28(3), 274-280. doi:10.1002/hup.2320

Ansari, T. L., \& Derakshan, N. (2010). Anxiety impairs inhibitory control but not volitional action control. Cogn Emot, 24(2), 241-254. doi:10.1080/02699930903381531

Ansari, T. L., \& Derakshan, N. (2011a). The neural correlates of cognitive effort in anxiety: effects on processing efficiency. Biol Psychol, 86(3), 337-348. doi:10.1016/j.biopsycho.2010.12.013

Ansari, T. L., \& Derakshan, N. (2011b). The neural correlates of impaired inhibitory control in anxiety. Neuropsychologia, 49(5), 1146-1153. doi:10.1016/j.neuropsychologia.2011.01.019

Ansari, T. L., Derakshan, N., \& Richards, A. (2008). Effects of anxiety on task switching: Evidence from the mixed antisaccade task. Cognitive, Affective, \& Behavioral Neuroscience, 8(3), 229-238. doi:10.3758/cabn.8.3.229

Bar-Haim, Y., Lamy, D., Pergamin, L., Bakermans-Kranenburg, M. J., \& van, I. M. H. (2007). Threat-related attentional bias in anxious and nonanxious individuals: a metaanalytic study. Psychol Bull, 133(1), 1-24. doi:10.1037/0033-2909.133.1.1

Beatty, J. (1982). Task-evoked pupillary responses, processing load, and the structure of processing resources. Psychol Bull, 91(2), 276-292. doi:10.1037//0033-2909.91.2.276

Beatty, J., \& Lucero-Wagoner, B. (2000). The Pupillary System. In J. T. Cacioppo, L. G. Tassinary, \& G. G. Bernston (Eds.), Handbook of Psychophysiology (pp. 142-162). NY: Cambridge University Press. 
Berggren, N., \& Derakshan, N. (2013). Attentional control deficits in trait anxiety: why you see them and why you don't. Biol Psychol, 92(3), 440-446. doi:10.1016/j.biopsycho.2012.03.007

Berggren, N., Richards, A., Taylor, J., \& Derakshan, N. (2013). Affective attention under cognitive load: reduced emotional biases but emergent anxiety-related costs to inhibitory control. Front Hum Neurosci, 7, 188. doi:10.3389/fnhum.2013.00188

Bishop, S. J. (2009). Trait anxiety and impoverished prefrontal control of attention. Nat Neurosci, 12(1), 92-98. doi:10.1038/nn.2242

Bradley, M. M., Miccoli, L., Escrig, M. A., \& Lang, P. J. (2008). The pupil as a measure of emotional arousal and autonomic activation. Psychophysiology, 45(4), 602-607. doi:10.1111/j.1469-8986.2008.00654.x

Browning, M., Behrens, T. E., Jocham, G., O'Reilly, J. X., \& Bishop, S. J. (2015). Anxious individuals have difficulty learning the causal statistics of aversive environments. Nat Neurosci, 18(4), 590-596. doi:10.1038/nn.3961

Bruin, K. J., \& Wijers, A. A. (2002). Inhibition, response mode, and stimulus probability: a comparative event-related potential study. Clin Neurophysiol, 113(7), 1172-1182. doi:10.1016/S1388-2457(02)00141-4

Curtis, C. E., \& D'Esposito, M. (2003). Persistent activity in the prefrontal cortex during working memory. Trends in Cognitive Sciences, 7(9), 415-423. doi:10.1016/s13646613(03)00197-9

Donders, F. C. (1969). On the speed of mental processes. Acta Psychol (Amst), 30, 412-431. doi:10.1016/0001-6918(69)90065-1

Eimer, M. (1993). Effects of attention and stimulus probability on ERPs in a Go/Nogo task. Biol Psychol, 35(2), 123-138. doi:10.1016/0301-0511(93)90009-W 
Eysenck, M. W., \& Derakshan, N. (2011). New perspectives in attentional control theory. Personality and Individual Differences, 50(7), 955-960. doi:10.1016/j.paid.2010.08.019

Eysenck, M. W., Derakshan, N., Santos, R., \& Calvo, M. G. (2007). Anxiety and cognitive performance: attentional control theory. Emotion, 7(2), 336-353. doi:10.1037/15283542.7.2.336

Falkenstein, M., Hoormann, J., \& Hohnsbein, J. (1999). ERP components in Go Nogo tasks and their relation to inhibition. Acta Psychologica, 101(2-3), 267-291. doi:10.1016/S0001-6918(99)00008-6

Ford, K. A., Goltz, H. C., Brown, M. R., \& Everling, S. (2005). Neural processes associated with antisaccade task performance investigated with event-related FMRI. Journal of Neurophysiology, 94(1), 429-440. doi:10.1152/jn.00471.2004

Garner, M., Attwood, A., Baldwin, D. S., James, A., \& Munafo, M. R. (2011). Inhalation of $7.5 \%$ carbon dioxide increases threat processing in humans. Neuropsychopharmacology, 36(8), 1557-1562. doi:10.1038/npp.2011.15

Geva, R., Zivan, M., Warsha, A., \& Olchik, D. (2013). Alerting, orienting or executive attention networks: differential patters of pupil dilations. Front Behav Neurosci, 7, 145. doi:10.3389/fnbeh.2013.00145

Granholm, E., Morris, S., Asarnow, R. F., Chock, D., \& Jeste, D. V. (2000). Accelerated agerelated decline in processing resources in schizophrenia: evidence from pupillary responses recorded during the span of apprehension task. J Int Neuropsychol Soc, 6(1), 30-43. doi:10.1017/s 1355617700611049

Hepsomali, P., Hadwin, J. A., Liversedge, S. P., \& Garner, M. (2017). Pupillometric and saccadic measures of affective and executive processing in anxiety. Biol Psychol, 127, 173-179. doi:10.1016/j.biopsycho.2017.05.013 
Herrmann, M. J., Jacob, C., Unterecker, S., \& Fallgatter, A. J. (2003). Reduced responseinhibition in obsessive-compulsive disorder measured with topographic evoked potential mapping. Psychiatry Res, 120(3), 265-271. doi:10.1016/s01651781(03)00188-4

Kahneman, D., \& Beatty, J. (1966). Pupil diameter and load on memory. Science, 154(3756), 1583-1585. doi:10.1126/science.154.3756.1583

Karatekin, C., Marcus, D. J., \& Couperus, J. W. (2007). Regulation of cognitive resources during sustained attention and working memory in 10-year-olds and adults. Psychophysiology, 44(1), 128-144. doi:10.1111/j.1469-8986.2006.00477.x

Khader, P., Schicke, T., Roder, B., \& Rosler, F. (2008). On the relationship between slow cortical potentials and BOLD signal changes in humans. Int J Psychophysiol, 67(3), 252-261. doi:10.1016/j.ijpsycho.2007.05.018

Kim, M. S., Kim, Y. Y., Yoo, S. Y., \& Kwon, J. S. (2007). Electrophysiological correlates of behavioral response inhibition in patients with obsessive-compulsive disorder. Depress Anxiety, 24(1), 22-31. doi:10.1002/da.20195

Lavric, A., Pizzagalli, D. A., \& Forstmeier, S. (2004). When 'go' and 'nogo' are equally frequent: ERP components and cortical tomography. Eur J Neurosci, 20(9), 24832488. doi:10.1111/j.1460-9568.2004.03683.x

Low, K. A., \& Miller, J. (1999). The usefulness of partial information: effects of go probability in the choice/Nogo task. Psychophysiology, 36(3), 288-297. doi:10.1017/s0048577299980332

Luna, B., \& Velanova, K. (2011). Development from reflexive to controlled eye movements. In S. P. Liversedge, I. D. Gilchrist, \& S. Everling (Eds.), The Oxford Handbook of Eye Movements (pp. 621-642). NY: Oxford University Press. 
Mandrick, K., Peysakhovich, V., Rémy, F., Lepron, E., \& Causse, M. (2016). Neural and psychophysiological correlates of human performance under stress and high mental workload. Biological Psychology, 121, 62-73.

Najmi, S., Amir, N., Frosio, K. E., \& Ayers, C. (2015). The effects of cognitive load on attention control in subclinical anxiety and generalised anxiety disorder. Cogn Emot, 29(7), 1210-1223. doi:10.1080/02699931.2014.975188

Oldfield, R. C. (1971). The assessment and analysis of handedness: The Edinburgh inventory. Neuropsychologia, 9(1), 97-113. doi:10.1016/0028-3932(71)90067-4

Pacheco-Unguetti, A. P., Acosta, A., Callejas, A., \& Lupianez, J. (2010). Attention and anxiety: different attentional functioning under state and trait anxiety. Psychol Sci, 21(2), 298-304. doi:10.1177/0956797609359624

Partala, T., \& Surakka, V. (2003). Pupil size variation as an indication of affective processing. International Journal of Human-Computer Studies, 59(1-2), 185-198. doi:10.1016/S1071-5819(03)00017-X

Righi, S., Mecacci, L., \& Viggiano, M. P. (2009). Anxiety, cognitive self-evaluation and performance: ERP correlates. J Anxiety Disord, 23(8), 1132-1138. doi:10.1016/j.janxdis.2009.07.018

Roche, R. A., Garavan, H., Foxe, J. J., \& O'Mara, S. M. (2005). Individual differences discriminate event-related potentials but not performance during response inhibition. Exp Brain Res, 160(1), 60-70. doi:10.1007/s00221-004-1985-z

Rämä, P., Carlson, S., Kekomi, J., \& Hämäläinen, H. (1995). A spatial oculomotor memorytask performance priduces a task-related slow shift in human electroencaphalography. Electroencephalography and clinical Neurophysiology, 94, 371-380. 
Sehlmeyer, C., Konrad, C., Zwitserlood, P., Arolt, V., Falkenstein, M., \& Beste, C. (2010). ERP indices for response inhibition are related to anxiety-related personality traits. Neuropsychologia, 48(9), 2488-2495. doi:10.1016/j.neuropsychologia.2010.04.022

Simmonds, D. J., Pekar, J. J., \& Mostofsky, S. H. (2008). Meta-analysis of Go/No-go tasks demonstrating that fMRI activation associated with response inhibition is taskdependent. Neuropsychologia, 46(1), 224-232. doi:10.1016/j.neuropsychologia.2007.07.015

Spielberger, C. D., Gorusch, R. L., Lushene, R., Vagg, P. R., \& Jacobs, G. A. (1983). Manual for State-Trait Anxiety Inventory. Palo Alto, CA: Consulting Psychologists Press.

Studer, P., Kratz, O., Gevensleben, H., Rothenberger, A., Moll, G. H., Hautzinger, M., \& Heinrich, H. (2014). Slow cortical potential and theta/beta neurofeedback training in adults: effects on attentional processes and motor system excitability. Front Hum Neurosci, 8, 555. doi:10.3389/fnhum.2014.00555

Turner, L. M., Croft, R. J., Churchyard, A., Looi, J. C., Apthorp, D., \& Georgiou-Karistianis, N. (2015). Abnormal Electrophysiological Motor Responses in Huntington's Disease: Evidence of Premanifest Compensation. PLoS One, 10(9), e0138563. doi:10.1371/journal.pone.0138563

Vytal, K. E., Cornwell, B. R., Letkiewicz, A. M., Arkin, N. E., \& Grillon, C. (2013). The complex interaction between anxiety and cognition: insight from spatial and verbal working memory. Frontiers in Human Neuroscience, 7(93), 1-11. doi:10.3389/Fnhum.2013.00093

Walter, W. G., Cooper, R., Aldridge, V. J., McCallum, W. C., \& Winter, A. L. (1964). Contingent Negative Variation : An Electric Sign of Sensori-Motor Association and Expectancy in the Human Brain. Nature, 203(4943), 380-384. doi:10.1038/203380a0 
Wilson, E., \& MacLeod, C. (2003). Contrasting two accounts of anxiety-linked attentional bias: selective attention to varying levels of stimulus threat intensity. $J$ Abnorm Psychol, 112(2), 212-218. doi:10.1037/0021-843x.112.2.212

Yang, L., \& Li, X. B. (2014). Anxiety alters brain activity of response inhibition: evidence from event-related potentials and source current density analysis. 2014 4th IEEE International Conference on Information Science and Technology (Icist), 160-163. doi:10.1109/icist.2014.6920355 


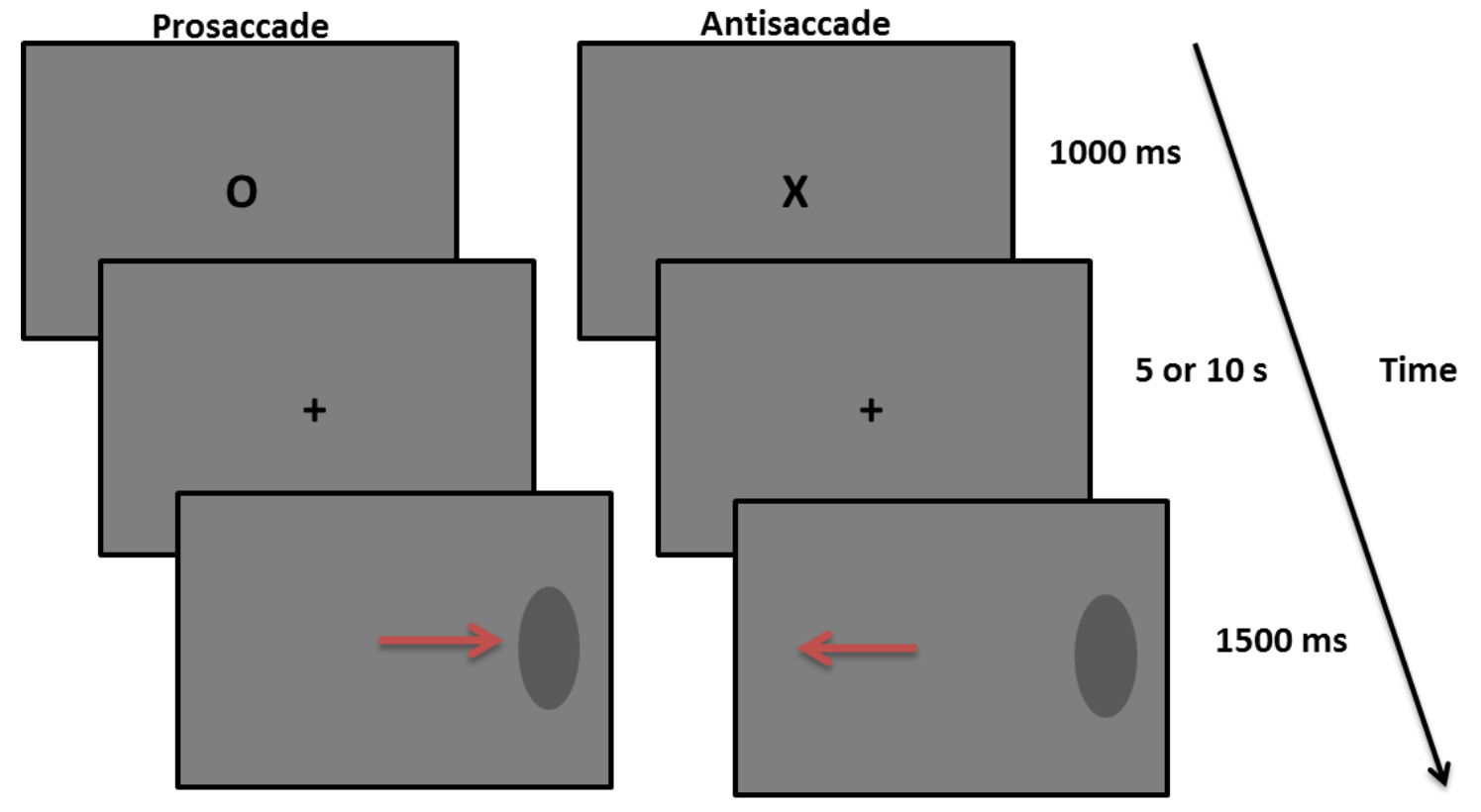

Figure 1. Flowchart of prosaccade and antisaccade trials in short and long delay trials. 

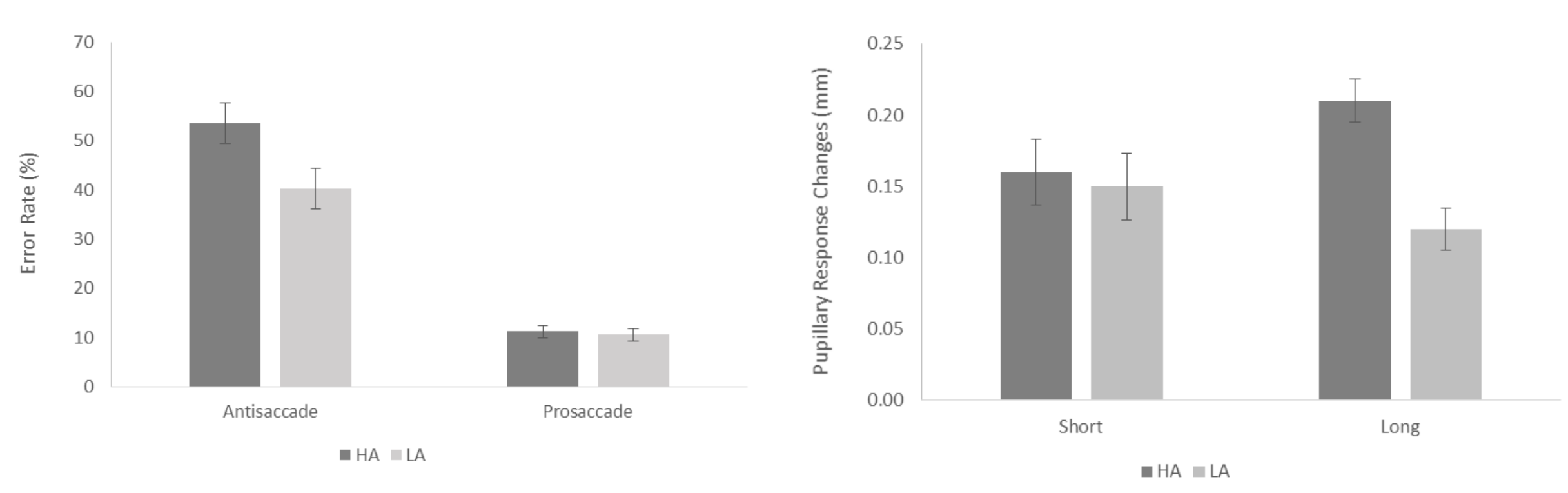

$\mathrm{Fz}$
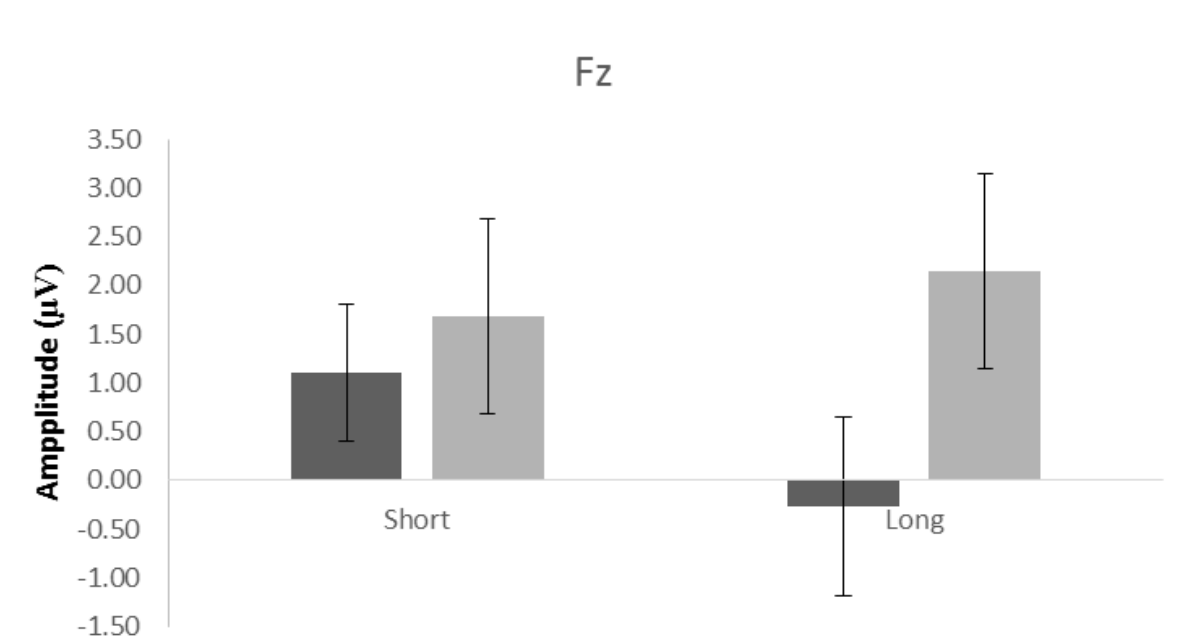

- HA $=$ LA

Figure 2. Percentage of saccade error in high-anxious (HA) and low-anxious (LA) groups on each of the antisaccade and prosaccade trials (top left panel), Peak pupillary response changes during long and short delay trials in high anxious (HA) and low anxious (LA) groups (top right panel), Mean CNV activity at Fz during short and long delay in high (HA) and low (LA) anxious individuals (bottom panel) (bars represent standard errors). 


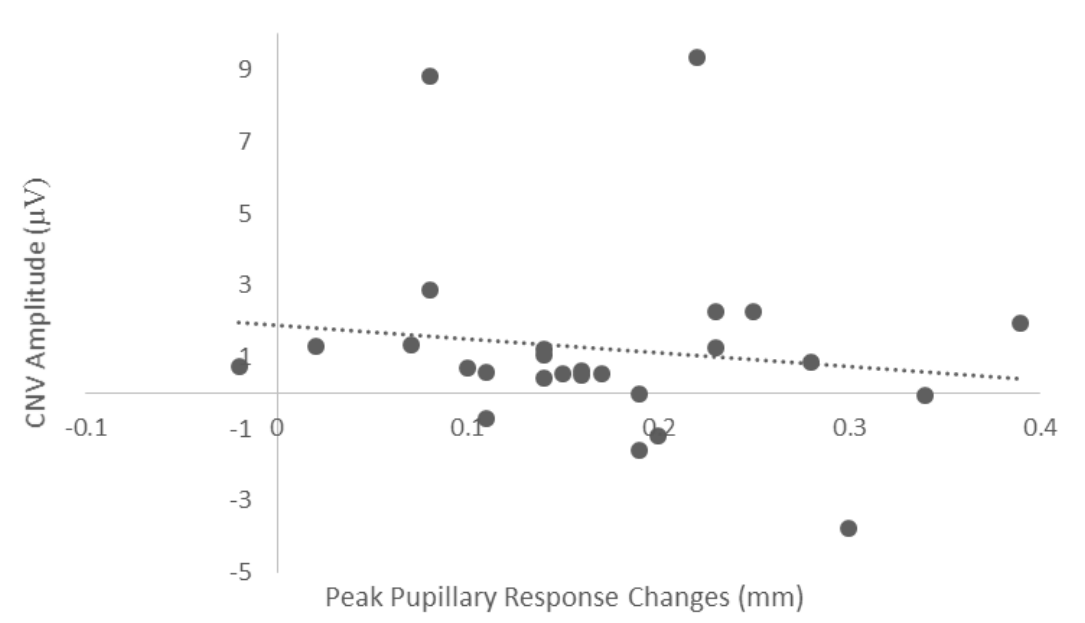

Figure 3. Scatterplot of the relation between CNV and pupillary responses.
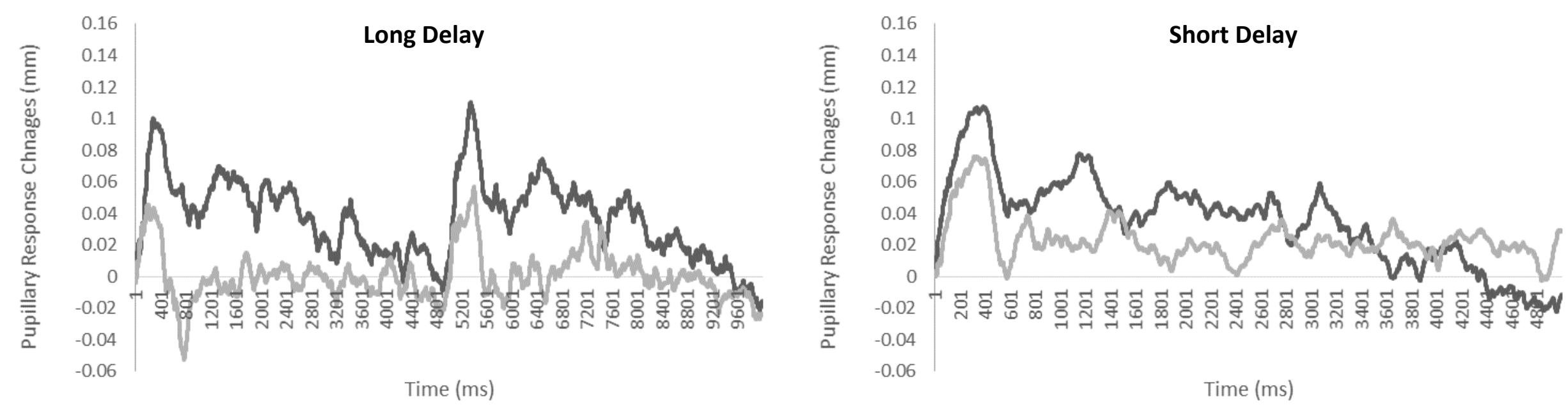

$-\mathrm{HA}-\mathrm{LA}$

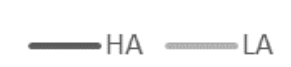

Figure 4. Pupillary response time series in high-anxious (HA) and low-anxious (LA) groups during long (left hand panel), and short (right hand panel) delay trials. 

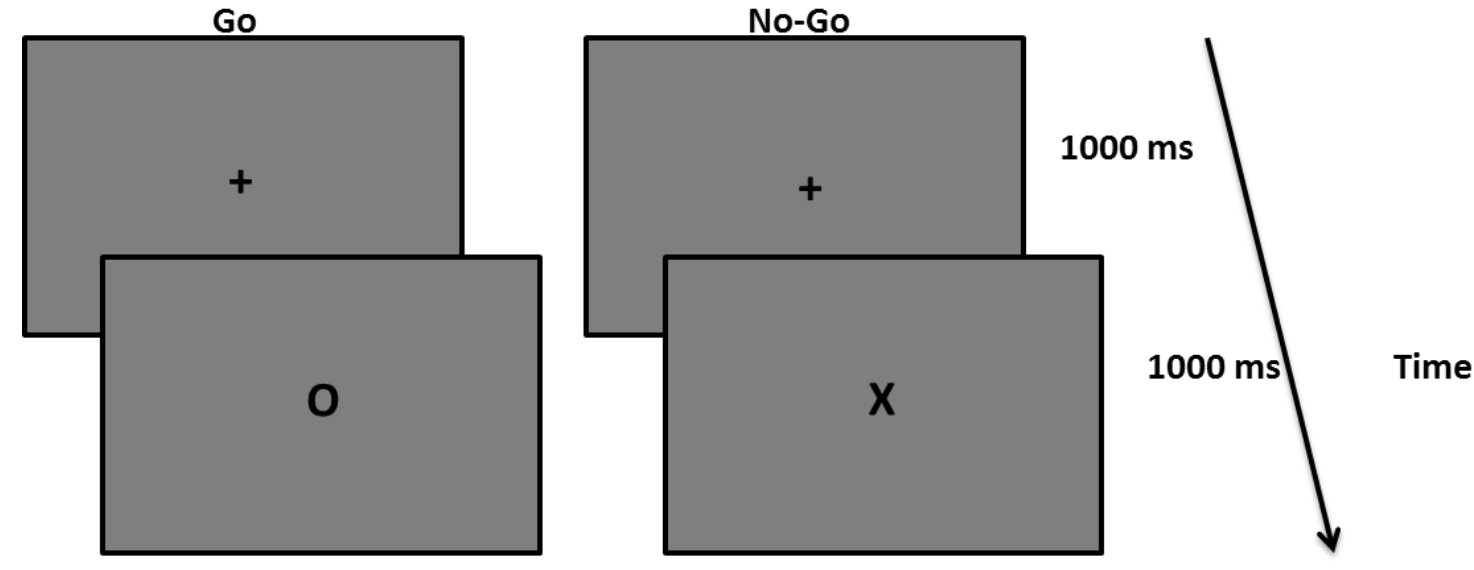

Figure 5. Flowchart of Go and No-Go trials 
Go

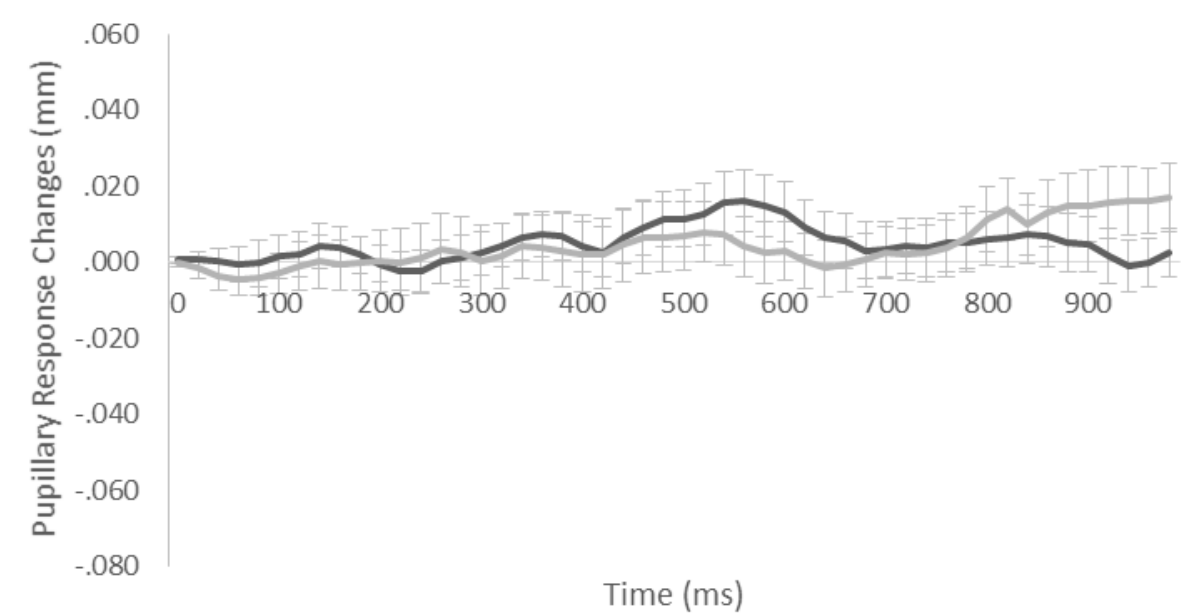

No-Go

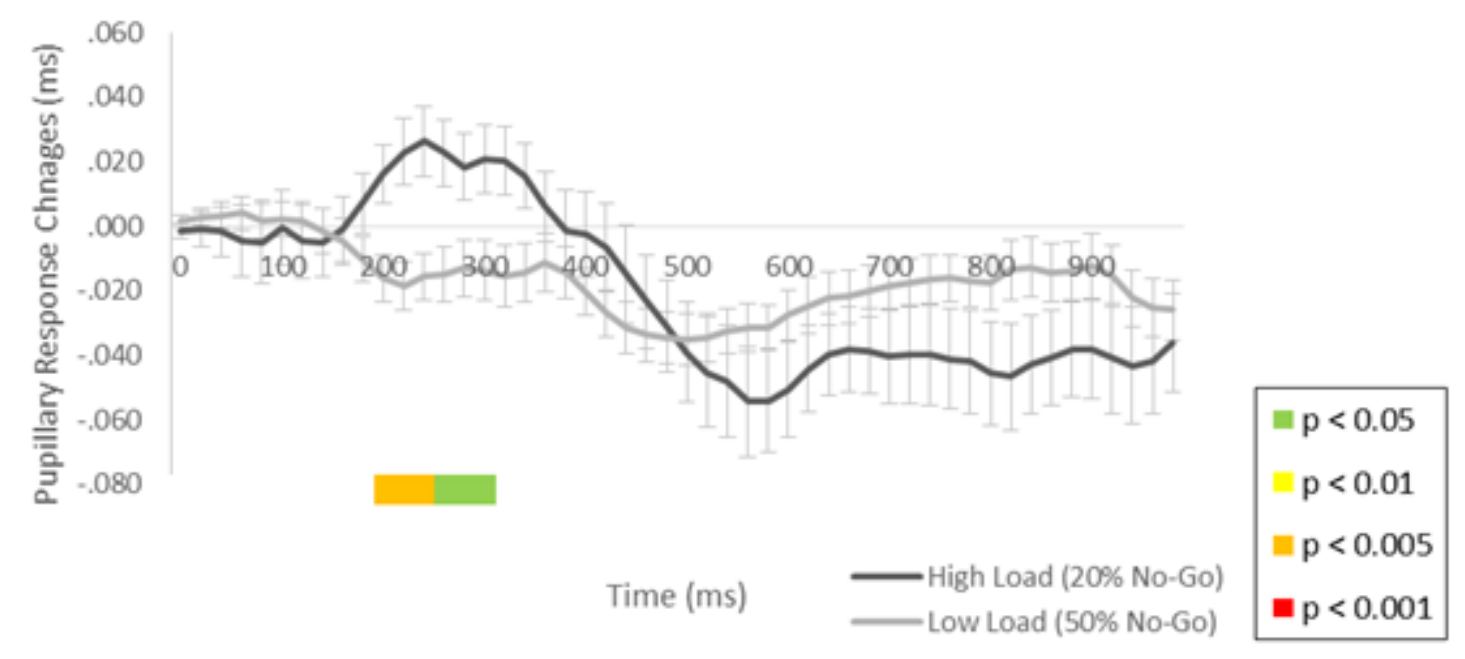

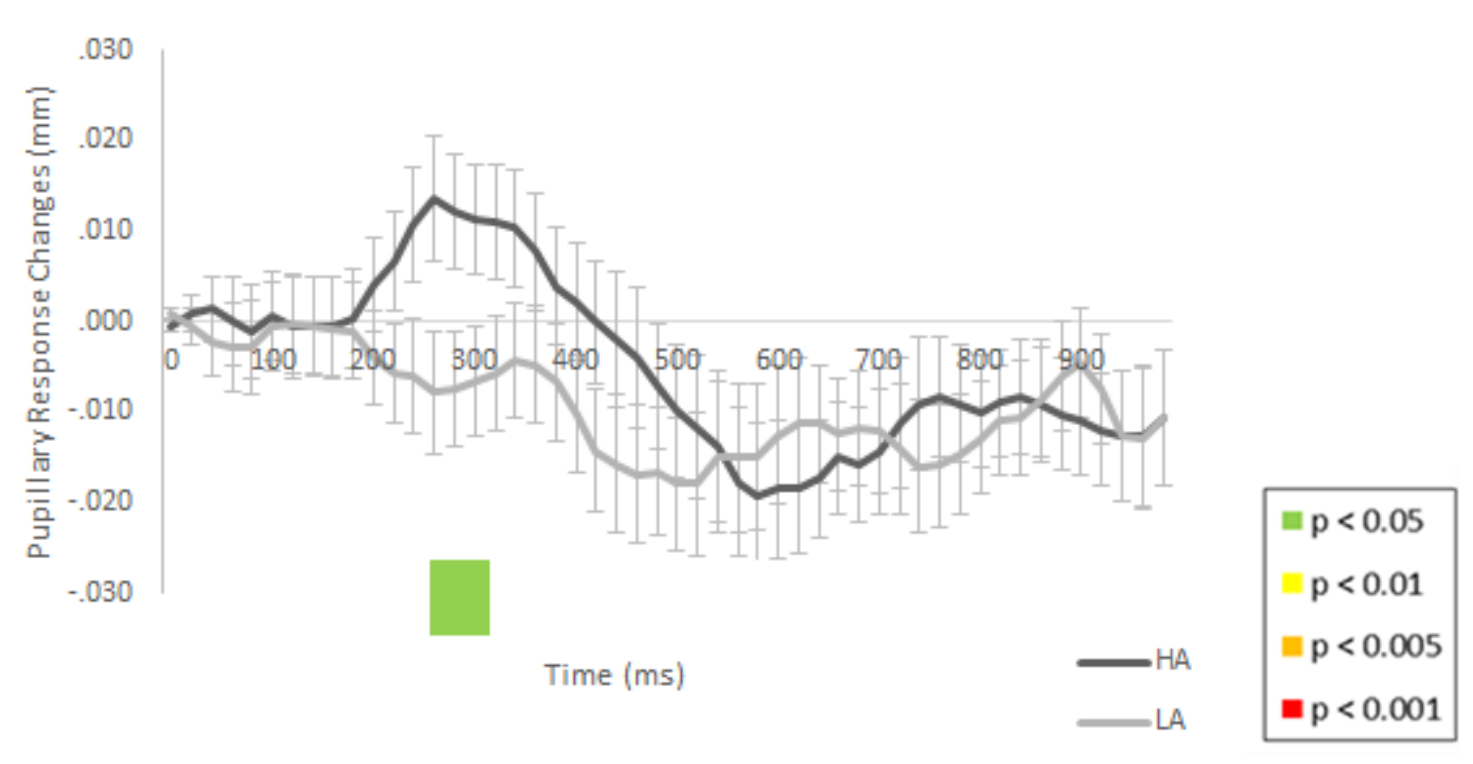

Figure 6. Pupillary response time series during Go trials (top left panel) and No-Go trials (top right panel) under high and low load, and pupillary response time series in high-anxious (HA) and low-anxious (LA) groups (bottom panel) (in all figures bars represent standard errors). 

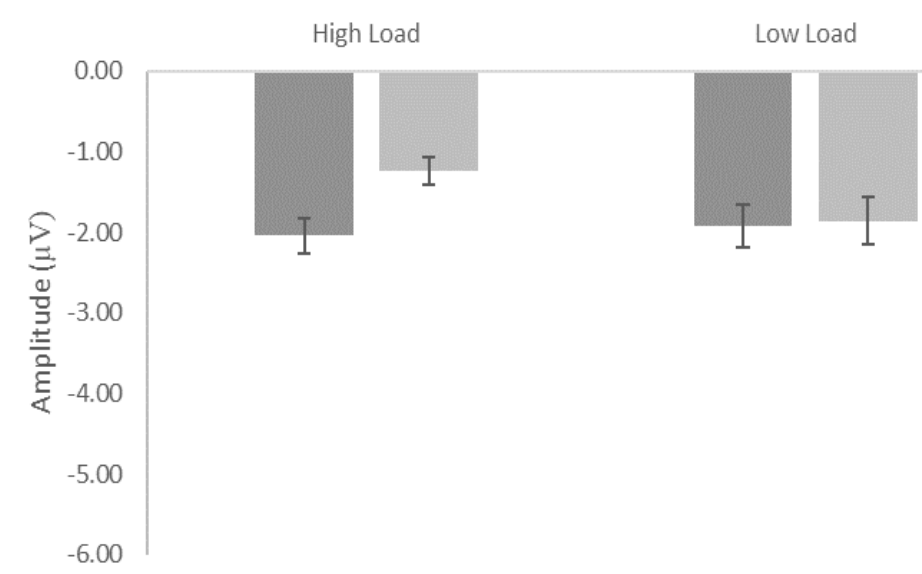

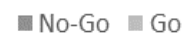

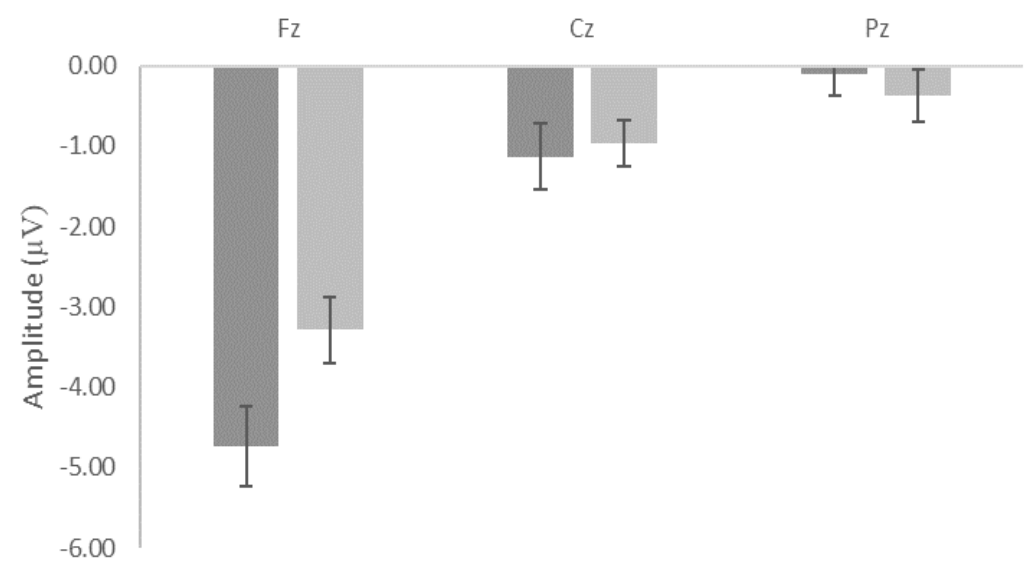

- No-Go $=\mathrm{Go}$

Figure 7. Mean N2 amplitudes during No-Go and Go trials under high and low load conditions (left hand panel), and mean N2 amplitudes during No-Go and Go trials at $\mathrm{Fz}, \mathrm{Cz}$, and, $\mathrm{Pz}$ (right hand panel) (in all figures bars represent standard errors).

LA

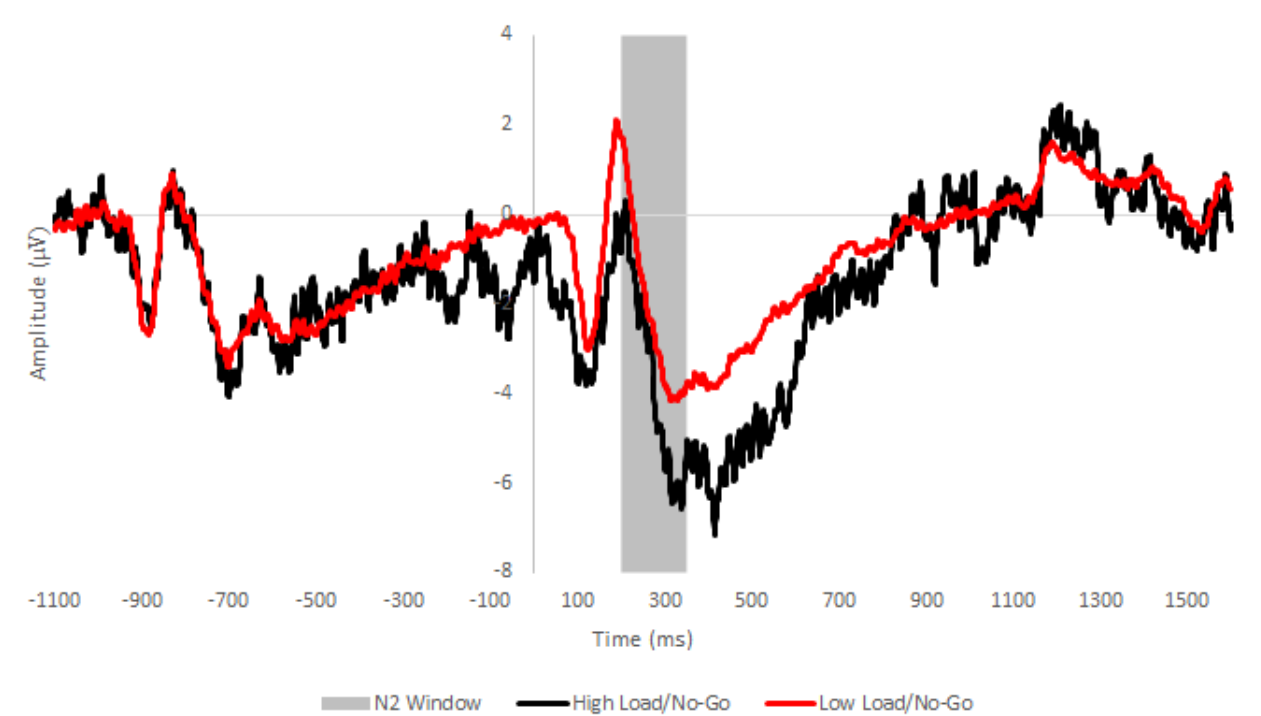

HA

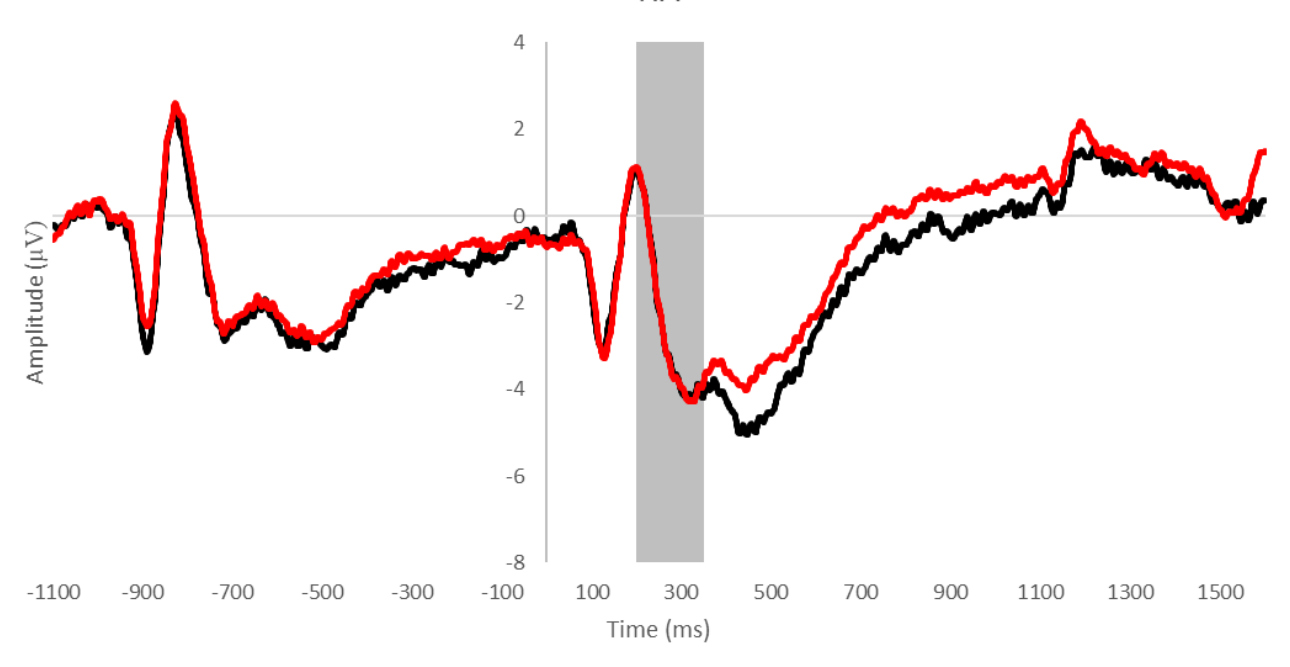

Figure 8. Grand average waveforms of frontal N2 amplitudes in No-Go trials under high and low load conditions in low-anxious (LA) (left hand panel) and high-anxious (HA) (right hand panel) groups. (shaded area represents the critical period of interest). 